\title{
Impact of Local Lattice Disorder on Spin and Orbital Orders in $\mathrm{Ca}_{2-x} \mathrm{Sr}_{x} \mathrm{RuO}_{4}$
}

\author{
Takuya Sugimoto ${ }^{1}$, Daiki Ootsuki², Takashi Mizokawa ${ }^{1,2}$ \\ ${ }^{1}$ Department of Complexity Science and Engineering, University of Tokyo, 5-1-5 \\ Kashiwanoha, Kashiwa, Chiba 277-8561, Japan \\ ${ }^{2}$ Department of Physics, University of Tokyo, 5-1-5 Kashiwanoha, Kashiwa, Chiba \\ 277-8561, Japan
}

\begin{abstract}
We have studied relationship between local lattice disorder, $\mathrm{Ru} 4 d$ spin-orbit interaction, and global spin/orbital orders of $\mathrm{Ca}_{2-x} \mathrm{Sr}_{x} \mathrm{RuO}_{4}$ in the Sr-rich and Ca-rich regions of its phase diagram by using unrestricted Hartree-Fock calculation on a $8 \times 8 \mathrm{RuO}_{4}$ lattice model. The calculations show that the local elongation of $\mathrm{RuO}_{6}$ octahedron in an antiferromagnetic insulator $\mathrm{Ca}_{2} \mathrm{RuO}_{4}$ induces local orbital change with making the Mott gap narrower. On the other hand, local compression of $\mathrm{RuO}_{6}$ octahedron in a paramagnetic metal $\mathrm{Sr}_{2} \mathrm{RuO}_{4}$ induces global antiferromagnetic or ferromagnetic states. This result is consistent with a recent systematic $\mu \mathrm{SR}$ study by Carlo et al. which has revealed the static antiferromagnetic order at low temperature in the Sr-rich region. KEYWORDS: layered ruthenates, antiferromagnetism, orbital degeneracy, Mott insulator
\end{abstract}




\section{Introduction}

The layered perovskite $\mathrm{Ca}_{2-x} \mathrm{Sr}_{x} \mathrm{RuO}_{4}$ (CSRO) system has been attracting considerable interest due to the interesting evolution from the spin-triplet superconducting state in $\mathrm{Sr}_{2} \mathrm{RuO}_{4}$ to the Mott insulating state in $\mathrm{Ca}_{2} \mathrm{RuO}_{4} \cdot{ }^{1-4}$ The structural phase diagram of CSRO exhibits an interesting interplay between titling, rotation, and Jahn-Teller distortion of $\mathrm{RuO}_{6}$ octahedron. ${ }^{5}$ The magnetic and electronic properties of CSRO strongly correlate with the structural distortions. The Mott transition at $x=0.0\left(\mathrm{Ca}_{2} \mathrm{RuO}_{4}\right)$ is accompanied by the $\mathrm{Ru} 4 d$ orbital change due to the Jahn-Teller distortion. ${ }^{6,7}$ For $x \leq 0.2$, CSRO is an antiferromagnetic insulator at low temperature due to the JahnTeller driven compression of $\mathrm{RuO}_{6}$ octahedron along the $c$-axis. For $0.2 \leq x \leq 0.5$, the tilting of $\mathrm{RuO}_{6}$ octahedron provides orthorhombic distortion, and the magnetic susceptibility shows a heavy Fermion behavior at low temperature. The orthorhombic distortion seems to suppress the ferromagnetic and/or small- $q$ antiferromagnetic fluctuation while it still enhances the mass renormalization towards $x=0.2$. Although the mass enhancement around $x=0.2$ is claimed to be explained by orbital selective Mott transition, ${ }^{8}$ existence of orbital selective Mott transition in multi-band Hubbard models depends on the details of parameters in the Hubbard Hamiltonians. ${ }^{9,10}$ It is still controversial whether the orbital selective Mott transition of the multi-band $\mathrm{Ru} 4 d$ electrons is relevant for the electronic phase diagram of CSRO or not. As for the tiny Sr substitution in the Mott insulating state of $\mathrm{Ca}_{2} \mathrm{RuO}_{4}$, the transport properties are dramatically changed by the Sr doping. ${ }^{11}$

Very recently, a systematic $\mu \mathrm{SR}$ study has revealed that static antiferromagnetic order exists at low temperature even in the Sr-rich region $(1.5 \leq x \leq 2.0)$ of its phase diagram. ${ }^{12}$ This indicates that the local distortion of $\mathrm{RuO}_{6}$ octahedron introduced by the Ca substitution plays important roles to stabilize the antiferromagnetic state. On the other hand, in the Ca-rich region $(0.0 \leq x \leq 0.5)$, the Sr-substitution reduces the magnitude of the Jahn-Teller distortion, tilting, and rotation of the $\mathrm{RuO}_{6}$ octahedron in $\mathrm{Ca}_{2} \mathrm{RuO}_{4}$ and, consequently, destroys the spin and orbital orders of $\mathrm{Ca}_{2} \mathrm{RuO}_{4}$. In order to gain deeper understandings of the phase diagram, it is important to study the $\mathrm{Ru} 4 d$ spin-orbital states using a realistic model in which the effects of spin-orbit interaction and lattice distortions are considered.

In this paper, we investigate the $\mathrm{Sr}$ or $\mathrm{Ca}$ doping effects on the electronic structure of CSRO system at both ends of its phase diagram (which are $\mathrm{Sr}_{2} \mathrm{RuO}_{4}$ and $\mathrm{Ca}_{2} \mathrm{RuO}_{4}$ ) 
by means of unrestricted Hartree-Fock (HF) calculation, which includes the spin-orbit interaction and lattice distortion induced by the chemical substitution.

\section{Method of calculation}

We use the multiband $d-p$ model where full degeneracy of the $\mathrm{Ru} 4 d$ orbitals and the $\mathrm{O} 2 p$ orbitals are taken into account. The Hamiltonian is given by

$$
\begin{aligned}
\hat{\mathscr{H}}= & \hat{\mathscr{H}}_{p}+\hat{\mathscr{H}}_{d}+\hat{\mathscr{H}}_{p d} \\
\hat{\mathscr{H}}_{p}= & \sum_{k l \sigma} \epsilon_{k}^{p} p_{k l \sigma}^{\dagger} p_{k l \sigma}+\sum_{k l l^{\prime} \sigma} V_{k l l^{\prime}}^{p p} p_{k l \sigma}^{\dagger} p_{k l^{\prime} \sigma}+\text { h.c. } \\
\hat{\mathscr{H}}_{d}= & \epsilon_{d}^{0} \sum_{i \alpha m \sigma} d_{i \alpha m \sigma}^{\dagger} d_{i \alpha m \sigma}+\sum_{i \alpha m m^{\prime} \sigma \sigma^{\prime}} h_{m m^{\prime} \sigma \sigma^{\prime}} d_{i \alpha m \sigma}^{\dagger} d_{i \alpha m^{\prime} \sigma^{\prime}} \\
& +u \sum_{i \alpha m} d_{i \alpha m \uparrow}^{\dagger} d_{i \alpha m \uparrow} d_{i \alpha m \downarrow}^{\dagger} d_{i \alpha m \downarrow} \\
& +u^{\prime} \sum_{i \alpha m m^{\prime}} d_{i \alpha m \uparrow}^{\dagger} d_{i \alpha m \uparrow} d_{i \alpha m \downarrow}^{\dagger} d_{i \alpha m \downarrow} \\
& +\left(u^{\prime}-j\right) \sum_{i \alpha m m^{\prime} \sigma} d_{i \alpha m \sigma}^{\dagger} d_{i \alpha m \sigma} d_{i \alpha m^{\prime} \sigma}^{\dagger} d_{i \alpha m^{\prime} \sigma} \\
& +j \sum_{i \alpha m m^{\prime}} d_{i \alpha m \uparrow}^{\dagger} d_{i \alpha m^{\prime} \uparrow} d_{i \alpha m^{\prime} \downarrow}^{\dagger} d_{i \alpha m \downarrow} \\
& +j^{\prime} \sum_{i \alpha m m^{\prime}} d_{i \alpha m \uparrow}^{\dagger} d_{i \alpha m^{\prime} \uparrow} d_{i \alpha m \downarrow}^{\dagger} d_{i \alpha m^{\prime} \downarrow} \\
\hat{\mathscr{H}}_{p d}= & \sum_{k m l \sigma} V_{k m l}^{p d} d_{k m \sigma}^{\dagger} p_{k l \sigma}+\mathrm{h} . c .
\end{aligned}
$$

Here, $d_{i \alpha m \sigma}^{\dagger}$ are creation operators for the $\mathrm{Ru} 4 d$ electrons at site $\alpha$ of the $i^{\text {th }}$ unit cell and $d_{k m \sigma}^{\dagger}$ and $p_{k l \sigma}^{\dagger}$ are creation operators for Bloch electrons which are constructed from the $m^{\text {th }}$ component of the $4 d$ orbitals and from the $l^{\text {th }}$ component of the $\mathrm{O} 2 p$ orbitals, respectively, with wave vector $\boldsymbol{k}$. The matrix $h_{m m^{\prime} \sigma \sigma^{\prime}}$ denotes the spin-orbit interaction and the effects of crystal field splitting. The magnitude of the spin-orbit interaction for the $\mathrm{Ru} 4 d$ orbital is fixed as $0.15 \mathrm{eV}$. The transfer integrals between the $\mathrm{O} 2 p$ orbitals $V_{k l l^{\prime}}^{p p}$ are given by Slater-Koster parameters $(p p \sigma)$ and $(p p \pi)$ which are fixed at $0.60 \mathrm{eV}$ and $-0.15 \mathrm{eV}$ respectively. The transfer integrals between the $\mathrm{Ru} 4 d$ and $\mathrm{O} 2 p$ orbitals $V_{k m l}^{p d}$ are represented by $(p d \pi)$ and $(p d \sigma)$. They are fixed as $(p d \sigma)=-2.8 \mathrm{eV}$ and $(p d \pi)=1.26 \mathrm{eV}$ for the longer in-plane $\mathrm{Ru}-\mathrm{O}$ bond of $\mathrm{Ca}_{2} \mathrm{RuO}_{4}$ whereas $(p d \sigma)=-3.4$ $\mathrm{eV}$ and $(p d \pi)=1.53 \mathrm{eV}$ for the shorter in-plane $\mathrm{Ru}-\mathrm{O}$ bond of $\mathrm{Sr}_{2} \mathrm{RuO}_{4}$. The summary of the material-dependent parameters are shown in Table I. The tilting of the $\mathrm{RuO}_{6}$ 
octahedron is included for $\mathrm{Ca}_{2} \mathrm{RuO}_{4}$. The distortion parameter $\delta_{\mathrm{JT}}$ is defined as $\delta_{\mathrm{JT}}=$ $d_{\text {apical }} / d_{\text {in-plane }}$ which is the ratio between the apical and in-plane Ru-O bond distances. The distortion parameter $\delta_{\text {JT }}$ is utilized to express the elongation/compression of $\mathrm{RuO}_{6}$ octahedron as in Fig. 4(a). In the Sr-rich (Ca-rich) region, $\delta_{\mathrm{JT}}=1.07$ (0.95) for the host lattice and $\delta_{\mathrm{JT}}=0.95$ (1.07) for the locally distorted site. When the $\mathrm{RuO}_{6}$ octahedron is distorted, the transfer integrals are scaled by Harrison's rule. The intra-atomic Coulomb interactions between $\mathrm{Ru} 4 d$ electrons are given by Kanamori parameters. They are fixed as $u=u^{\prime}+j+j^{\prime}=3.0 \mathrm{eV}$ and $j=j^{\prime}=0.5 \mathrm{eV}$. The charge-transfer energy $\Delta$ (fixed as $-0.4 \mathrm{eV})$ is defined by $\epsilon_{d}-\epsilon_{p}+4 U$, where $\epsilon_{d}$ and $\epsilon_{p}$ are the energies of the bare $\mathrm{Ru} 4 d$ and $\mathrm{O} 2 p$ orbitals and $U[=u-(20 / 9) j]$ is the multiplet-averaged $d-d$ Coulomb interaction fixed at $1.89 \mathrm{eV}$.

We set the $8 \times 8$ supercell with periodic boundary conditions and put the $\mathrm{Ru} 4 d$ and $\mathrm{O} 2 p$ electrons on its each site. The local distortion is introduced at one Ru site of the supercell as shown in Figure 4(b). The total number of electron in the supercell is 1792 (256 Ru 4d and 1536 O $2 p$ electrons). The HF mean-field treatment is applied to the two-body part in $\hat{\mathscr{H}}_{d}$ by replacing its average values, for instance,

$$
\begin{aligned}
& u \sum_{i \alpha m} d_{i \alpha m \uparrow}^{\dagger} d_{i \alpha m \uparrow} d_{i \alpha m \downarrow}^{\dagger} d_{i \alpha m \downarrow} \\
& \rightarrow \\
& \quad u \sum_{i \alpha m}\left\langle d_{i \alpha m \uparrow}^{\dagger} d_{i \alpha m \uparrow}\right\rangle d_{i \alpha m \downarrow}^{\dagger} d_{i \alpha m \downarrow} \\
& \quad+u \sum_{i \alpha m} d_{i \alpha m \uparrow}^{\dagger} d_{i \alpha m \uparrow}\left\langle d_{i \alpha m \downarrow}^{\dagger} d_{i \alpha m \downarrow}\right\rangle \\
& \quad-u \sum_{i \alpha m}\left\langle d_{i \alpha m \uparrow}^{\dagger} d_{i \alpha m \uparrow}\right\rangle\left\langle d_{i \alpha m \downarrow}^{\dagger} d_{i \alpha m \downarrow}\right\rangle
\end{aligned}
$$

In this Hartree-Fock calculation, we input the initial values of the order parameters such as $\left\langle d^{\dagger} d\right\rangle$ and diagonalize the mean-field Hamiltonian to get a set of eigen functions. Then the order parameters can be calculated using the obtained eigen functions. This self-consistency cycle is iterated until the successive difference of all the order parameters converge less than $10^{-4}$. 


\section{Results and Discussion}

We calculate the following four cases to see the doping effects. For the Ca-rich region, we calculate the orbital population of $\mathrm{Ca}_{2} \mathrm{RuO}_{4}$ and $\mathrm{Ca}_{2-x} \mathrm{Sr}_{x} \mathrm{RuO}_{4}$ with a single-site distortion by the tiny Sr doping (let us label these two cases as case (i) and (ii), respectively). For the Sr-rich region, we calculate $\mathrm{Sr}_{2} \mathrm{RuO}_{4}$ and $\mathrm{Ca}_{x} \mathrm{Sr}_{2-x} \mathrm{RuO}_{4}$ with a single-site distortion by the Ca doping (let us label these two cases as case (iii) and (iv), respectively). Since we set the $8 \times 8$ supercell, the ratio of one-site transposition is equal to $1 / 64 \simeq 0.0156$. In terms of the doping amount in this CSRO system, $1.56 \%$ is equivalent to $x=0.0312$ in $\mathrm{Ca}_{2-x} \mathrm{Sr}_{x} \mathrm{RuO}_{4}$ and $\mathrm{Ca}_{x} \mathrm{Sr}_{2-x} \mathrm{RuO}_{4}$, respectively.

\subsection{Ca-rich region}

The present $\mathrm{HF}$ calculation using the reasonable parameter set predicts that $\mathrm{Ca}_{2} \mathrm{RuO}_{4}$ is an antiferromagnetic insulator. The compression of the $\mathrm{RuO}_{6}$ octahedra stabilizes the $\mathrm{Ru} 4 d x y$ orbital compared to the $\mathrm{Ru} 4 d y z / z x$ orbitals (Jahn-Teller type energy splitting). The combination of the Jahn-Teller type energy splitting and the Ru $4 d$ spin-orbit interaction can provide an interesting magnetic anisotropy. The antiferromagnetic insulating state with the in-plane ( $x$ or $y$ axis) spin direction is lower in energy than that with the out-of-plane ( $z$ axis) spin direction. The energy difference is $37 \mathrm{meV}$ per Ru site for the present parameter set. The expectation value of $d^{\dagger} d$ for each orbital and spin component of the $\mathrm{Ru} 4 d t_{2 g}$ states for $\mathrm{Ca}_{2} \mathrm{RuO}_{4}$ [case (i)] is shown in Table II for the in-plane and out-of-plane antiferromagnetic states. In the out-of-plane case, the complex orbitals with type of $y z \pm i z x$ are unoccupied [namely, occupied by the two $\mathrm{Ru} 4 d t_{2 g}$ holes of the $\mathrm{Ru}^{4+}\left(d^{4}\right)$ configuration] while the $x y$ orbitals are almost fully occupied by the $\mathrm{Ru} 4 d t_{2 g}$ electrons. Consequently the orbital angular momentum along the $z$-axis is formed to align the spin moment along the $z$-axis. As for the in-plane case, the complex orbitals with type of $x y \pm i z x$ or $x y \pm i y z$ are unoccupied to give orbital angular momentum in the $x y$-plane.

Assuming that one of the $\mathrm{RuO}_{6}$ octahedra is elongated due to the tiny Sr doping in the case (ii), the orbital population of the elongated site would be affected due to the reverse of the Jahn-Teller energy splitting between the $x y$ and $y z / z x$ orbitals. Interestingly, the orbital population of the elongated site is dramatically changed for the in-plane antiferromagnetic state whereas the impact of the elongation is limited for the out-of-plane antiferromagnetic state. As shown in Table II, the orbital population of the elongated site is almost the same as that of the compressed sites for the out-of- 
plane antiferromagnetic state. On the other hand, the $x y$ orbitals accommodate more holes at the elongated site for the in-plane antiferromagnetic state. The impact of local reverse of the Jahn-Teller energy splitting strongly depends on the global spin direction due to the strong $\mathrm{Ru} 4 d$ spin-orbit coupling. Since the in-plane antiferromagnetic state is realized in $\mathrm{Ca}_{2} \mathrm{RuO}_{4}$, the present calculation indicates that the local elongation of the $\mathrm{RuO}_{6}$ octahedron produces the $\mathrm{Ru} 4 d x y$ hole which can affect the global spin and orbital orders of $\mathrm{Ca}_{2} \mathrm{RuO}_{4}$. The density of states (DOS) calculated for the cases (i) and (ii) (which are $\mathrm{Ca}_{2} \mathrm{RuO}_{4}$ and $\mathrm{Ca}_{2-x} \mathrm{Sr}_{x} \mathrm{RuO}_{4}$ at $x=0.0312$ ) with the in-plane antiferromagnetic states are shown in Figs. 4(a) and (b), respectively. One can see that the Mott gap of $\mathrm{Ca}_{2} \mathrm{RuO}_{4}$ becomes narrower by the local elongation of $\mathrm{RuO}_{6}$ octahedron $\left(\delta_{\mathrm{JT}}=1.07\right)$ induced by the tiny $\mathrm{Sr}$ doping. The reduction of the Mott gap is related to the local orbital change induced by the reverse of the Jahn-Teller energy splitting.

\subsection{Sr-rich region}

The present HF calculation using the reasonable parameter set predicts that $\mathrm{Sr}_{2} \mathrm{RuO}_{4}$ is a paramagnetic or ferromagnetic metal. For the present parameter set, the ferromagnetic metallic state is slightly lower in energy than the paramagnetic metallic state, and the energy difference is about $0.4 \mathrm{meV}$ per $\mathrm{Ru}$ site. In the HF approximation, stability of ferromagnetic or antiferromagnetic states tends to be overestimated. Therefore, the present calculation indicates that the parameter set is reasonable to analyze the paramagnetic metallic state of $\mathrm{Sr}_{2} \mathrm{RuO}_{4}$. The expectation value of $d^{\dagger} d$ for each orbital and spin component of the $\mathrm{Ru} 4 d t_{2 g}$ states for $\mathrm{Sr}_{2} \mathrm{RuO}_{4}$ [case (iii)] is shown in Table III for the paramagnetic metallic state and the ferromagnetic metallic state. In the paramagnetic state, the spin-up and spin-down states have the same population for the $x y, y x$, and $z x$ orbitals, respectively. In the ferromagnetic state, the spin polarization for the $y z / z x$ orbitals is larger than that of the $x y$ orbital, indicating that the $y z / z x$ states stabilized by the elongation of the $\mathrm{RuO}_{6}$ octahedra are playing important roles to provide the ferromagnetic interaction. As for the effect of tiny Ca doping to $\mathrm{Sr}_{2} \mathrm{RuO}_{4}$ [case (iv)], it is assumed that one of the $\mathrm{RuO}_{6}$ octahedra is compressed due to the $\mathrm{Ca}$ doping. The local compression of the $\mathrm{RuO}_{6}$ octahedron changes the stability of the paramagnetic state and the nature of the magnetic state. The paramagnetic metallic state becomes unstable and only the ferromagnetic and/or antiferromagnetic states are obtained as stable solutions. The $x y$ orbitals are more occupied at the compressed site to induce antiferromagnetic superexchange interaction. The global antiferromagnetic state 
is stabilized by the local orbital change. The spin direction is intermediate between the in-plane and out-of-plane directions probably due to the competition between the $\mathrm{Ru}$ $4 d$ spin-orbit interaction and the local Jahn-Teller splitting of the $\mathrm{Ru} 4 d$ levels. In the stable magnetic state, the in-plane components of the $\mathrm{Ru} 4 d$ spins are ferromagnetically aligned whereas the out-of-plane components are antiferromagnetically arranged. The DOS calculated for the cases (iii) and (iv) (which are $\mathrm{Sr}_{2} \mathrm{RuO}_{4}$ and $\mathrm{Ca}_{x} \mathrm{Sr}_{2-x} \mathrm{RuO}_{4}$ at $x=0.0312$ ) are shown in Figs. 4(a) and (b), respectively. The local compression of $\mathrm{RuO}_{6}$ octahedron by the tiny $\mathrm{Ca}$ doping induces the small band gap at the Fermi level, which can be assigned to the magnetic ordering.

\section{Conclusion}

Investigating the electronic structure of CSRO by unrestricted HF calculation, we find the effects of local distortions on spin and orbital orders of the Ru $4 d t_{2 g}$ states. As for the Ca-rich region, we find that the antiferromagnetism survives when the singlesite distortion in $\mathrm{Ca}_{2} \mathrm{RuO}_{4}$ does carry on. The orbital state is locally disturbed for the in-plane antiferromagnetic state resulting in the reduction of the Mott gap. As for the Sr-rich region, on the other hand, the single-site distortion in $\mathrm{Sr}_{2} \mathrm{RuO}_{4}$ changes the entire system to the ferromagnetic/antiferromagnetic state from the paramagnetic state. The present study captures the trend of effects on $\mathrm{Sr}_{2} \mathrm{RuO}_{4}$ by the tiny amount of Ca doping, which could explain the recent $\mu \mathrm{SR}$ study. ${ }^{12}$

\section{Acknowledgements}

The authors would like to thank Profs. Y. J. Uemura and N. L. Saini for fruitful discussions. 


\section{References}

1) Y. Maeno, H. Hashimoto, K. Yoshida, S. Nishizaki, T. Fujita, J. G. Bednorz, and F. Lichtenberg: Nature 372 (1994) 532.

2) S. Nakatsuji and Y. Maeno: Phys. Rev. Lett. 84 (2000) 2666.

3) S. Nakatsuji and Y. Maeno: Phys. Rev. B 62 (2000) 6458.

4) M. Imada, A. Fujimori, and Y. Tokura: Rev. Mod. Phys. 70 (1998) 1039.

5) O. Friedt, M. Braden, G. Andre, P. Adelmann, S. Nakatsuji, and Y. Maeno: Phys. Rev. B 63 (2001) 174432.

6) T. Mizokawa, L. H. Tjeng, G. A. Sawatzky, G. Ghiringhelli, O. Tjernberg, N. B. Brookes, H. Fukazawa, S. Nakatsuji, and Y. Maeno: Phys. Rev. Lett. 87 (2001) 077202 .

7) T. Mizokawa, L. H. Tjeng, H.-J. Lin, C. T. Chen, S. Schuppler, S. Nakatsuji, H. Fukazawa, and Y. Maeno: Phys. Rev. B 69 (2004) 132410.

8) V. I. Anisimov, I. A. Nekrasov, D. E. Kondakov, T. M. Rice, and M. Sigrist: Euro. Phys. J. B 25 (2002) 191.

9) A. Liebsch: Phys. Rev. Lett. 90 (2003) 096401.

10) A. Koga, N. Kawakami, T. M. Rice, and M. Sigrist: Phys. Rev. Lett. 92 (2004) 216402 .

11) S. Nakatsuji, V. Dobrosavljevic, D. Tanaskovic, M. Minakata, H. Fukazawa, and Y. Maeno: Phys. Rev. Lett. 93 (2004) 146401.

12) J. P. Carlo, T. Goko, I. M. Gat-Malureanu, P. L. Russo, A. T. Savici, A. A. Aczel, G. J. MacDougall, J. A. Rodriguez, T. J. Williams, G. M. Luke, C. R. Wiebe, Y. Yoshida, S. Nakatsuji, Y. Maeno, T. Taniguchi, and Y. J. Uemura: Nature Materials 11 (2012) 323.

13) T. Mizokawa and A. Fujimori: Phys. Rev. B 54 (1996) 5368. 
Tables:

Table I. Parameter sets for $\mathrm{Sr}_{2} \mathrm{RuO}_{4}$ and $\mathrm{Ca}_{2} \mathrm{RuO}_{4}$.

\begin{tabular}{cccc}
\hline \hline & $(p d \sigma)$ for in-plane Ru-O bond & tilting angle & $\delta_{\text {JT }}$ \\
\hline $\mathrm{Sr}_{2} \mathrm{RuO}_{4}$ & $-3.4 \mathrm{eV}$ & $0 \mathrm{deg}$. & 1.07 \\
$\mathrm{Ca}_{2} \mathrm{RuO}_{4}$ & $-2.8 \mathrm{eV}$ & $12.5 \mathrm{deg}$. & 0.95 \\
\hline
\end{tabular}

Table II. Expectation values of $\left\langle d_{m \sigma}^{\dagger} d_{m \sigma}>\right.$ with $m=x y, y z, z x$ and $\sigma=\uparrow, \downarrow$ in the Ru $4 d t_{2 g}$ states for case (i) and case (ii).

\begin{tabular}{c|cccccc}
\hline \hline case $(\mathrm{i}) \mathrm{Ca}_{2} \mathrm{RuO}_{4}$ & \multicolumn{7}{|c}{} \\
\hline in-plane $(x y) \mathrm{AFM}$ & $x y \uparrow$ & $x y \downarrow$ & $y z \uparrow$ & $y z \downarrow$ & $z x \uparrow$ & $z x \downarrow$ \\
& 0.95 & 0.95 & 0.53 & 0.76 & 0.52 & 0.77 \\
\hline out-of-plane $(z) \mathrm{AFM}$ & $x y \uparrow$ & $x y \downarrow$ & $y z \uparrow$ & $y z \downarrow$ & $z x \uparrow$ & $z x \downarrow$ \\
& 0.98 & 0.98 & 0.98 & 0.28 & 0.99 & 0.26 \\
\hline \hline case (ii) $\mathrm{Ca}_{2-x} \mathrm{Sr}_{x} \mathrm{RuO}_{4}$ & & & & & & \\
\hline in-plane $(x y) \mathrm{AFM}^{2}$ & $x y \uparrow$ & $x y \downarrow$ & $y z \uparrow$ & $y z \downarrow$ & $z x \uparrow$ & $z x \downarrow$ \\
compressed site $(\mathrm{Ca}$ site) & 0.97 & 0.96 & 0.64 & 0.64 & 0.64 & 0.64 \\
\hline in-plane $(x y) \mathrm{AFM}$ & $x y \uparrow$ & $x y \downarrow$ & $y z \uparrow$ & $y z \downarrow$ & $z x \uparrow$ & $z x \downarrow$ \\
elongated site $(\mathrm{Sr}$ site) & 0.66 & 0.66 & 0.64 & 0.64 & 0.96 & 0.96 \\
\hline out-of-plane $(z) \mathrm{AFM}$ & $x y \uparrow$ & $x y \downarrow$ & $y z \uparrow$ & $y z \downarrow$ & $z x \uparrow$ & $z x \downarrow$ \\
compressed site $(\mathrm{Ca}$ site) & 0.98 & 0.98 & 0.98 & 0.28 & 0.99 & 0.26 \\
\hline out-of-plane $(z) \mathrm{AFM}$ & $x y \uparrow$ & $x y \downarrow$ & $y z \uparrow$ & $y z \downarrow$ & $z x \uparrow$ & $z x \downarrow$ \\
elongated site (Sr site) & 0.99 & 0.98 & 0.99 & 0.28 & 0.99 & 0.26 \\
\hline \hline
\end{tabular}


Table III. Expectation values of $\left\langle d_{m \sigma}^{\dagger} d_{m \sigma}>\right.$ with $m=x y, y z, z x$ and $\sigma=\uparrow, \downarrow$ in the Ru $4 d t_{2 g}$ states for case (iii) and case (iv).

\begin{tabular}{c|cccccc}
\hline \hline case (iii) $\mathrm{Sr}_{2} \mathrm{RuO}_{4}$ & \multicolumn{1}{|c}{} & & & & \\
\hline $\mathrm{PM}$ & $x y \uparrow$ & $x y \downarrow$ & $y z \uparrow$ & $y z \downarrow$ & $z x \uparrow$ & $z x \downarrow$ \\
& 0.71 & 0.71 & 0.88 & 0.88 & 0.88 & 0.88 \\
\hline $\mathrm{FM}$ & $x y \uparrow$ & $x y \downarrow$ & $y z \uparrow$ & $y z \downarrow$ & $z x \uparrow$ & $z x \downarrow$ \\
& 0.67 & 0.77 & 0.80 & 0.94 & 0.80 & 0.94 \\
\hline \hline case (iv) $\mathrm{Ca}_{x} \mathrm{Sr}_{2-x} \mathrm{RuO}_{4}(\mathrm{Ca}-\mathrm{doped})$ & & & & & & \\
\hline $\mathrm{AFM}$ & $x y \uparrow$ & $x y \downarrow$ & $y z \uparrow$ & $y z \downarrow$ & $z x \uparrow$ & $z x \downarrow$ \\
elongated site (Sr site) & 0.60 & 0.85 & 0.85 & 0.88 & 0.86 & 0.87 \\
\hline $\mathrm{AFM}$ & $x y \uparrow$ & $x y \downarrow$ & $y z \uparrow$ & $y z \downarrow$ & $z x \uparrow$ & $z x \downarrow$ \\
compressed site (Ca site) & 0.79 & 0.94 & 0.61 & 0.89 & 0.61 & 0.89 \\
\hline FM & $x y \uparrow$ & $x y \downarrow$ & $y z \uparrow$ & $y z \downarrow$ & $z x \uparrow$ & $z x \downarrow$ \\
elongated site (Sr site) & 0.67 & 0.78 & 0.80 & 0.94 & 0.80 & 0.94 \\
\hline FM & $x y \uparrow$ & $x y \downarrow$ & $y z \uparrow$ & $y z \downarrow$ & $z x \uparrow$ & $z x \downarrow$ \\
compressed site (Ca site) & 0.88 & 0.91 & 0.68 & 0.81 & 0.68 & 0.81 \\
\hline \hline
\end{tabular}


Figure captions:

Figure 1:

(Color online) Schematic diagram of (a) $\mathrm{RuO}_{6}$ octahedron with the definition of $\delta_{\text {JT }}$ and (b) the supercell with a single-site distortion.

Figure 2:

(Color online) DOS (calculated with the $\mathrm{Ru} 4 d$ spin-orbit interaction) of (a) $\mathrm{Ca}_{2} \mathrm{RuO}_{4}$ and (b) $\mathrm{Ca}_{2-x} \mathrm{Sr}_{x} \mathrm{RuO}_{4}$ at $x=0.0312$.

Figure 3:

(Color online) DOS (calculated with the $\mathrm{Ru} 4 d$ spin-orbit interaction) of (a) $\mathrm{Sr}_{2} \mathrm{RuO}_{4}$ and (b) $\mathrm{Ca}_{x} \mathrm{Sr}_{2-x} \mathrm{RuO}_{4}$ at $x=0.0312$. 
(a) $\mathrm{Ru}$ Oxygeno

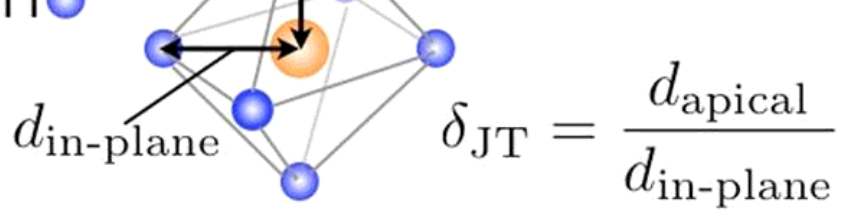

(b)

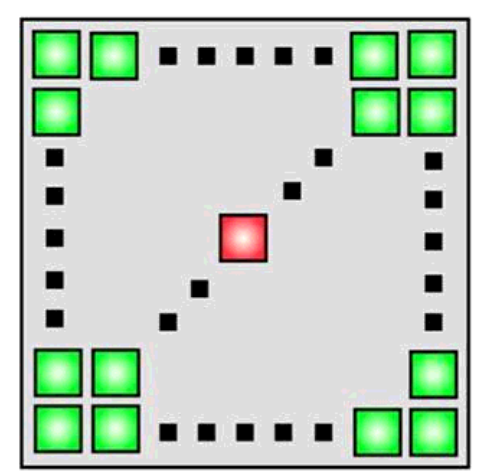

$\square \delta_{\mathrm{JT}}=1.07$

$\square \delta_{\mathrm{JT}}=0.95$ 
J. Phys. Soc. Jpn.

DRAFT
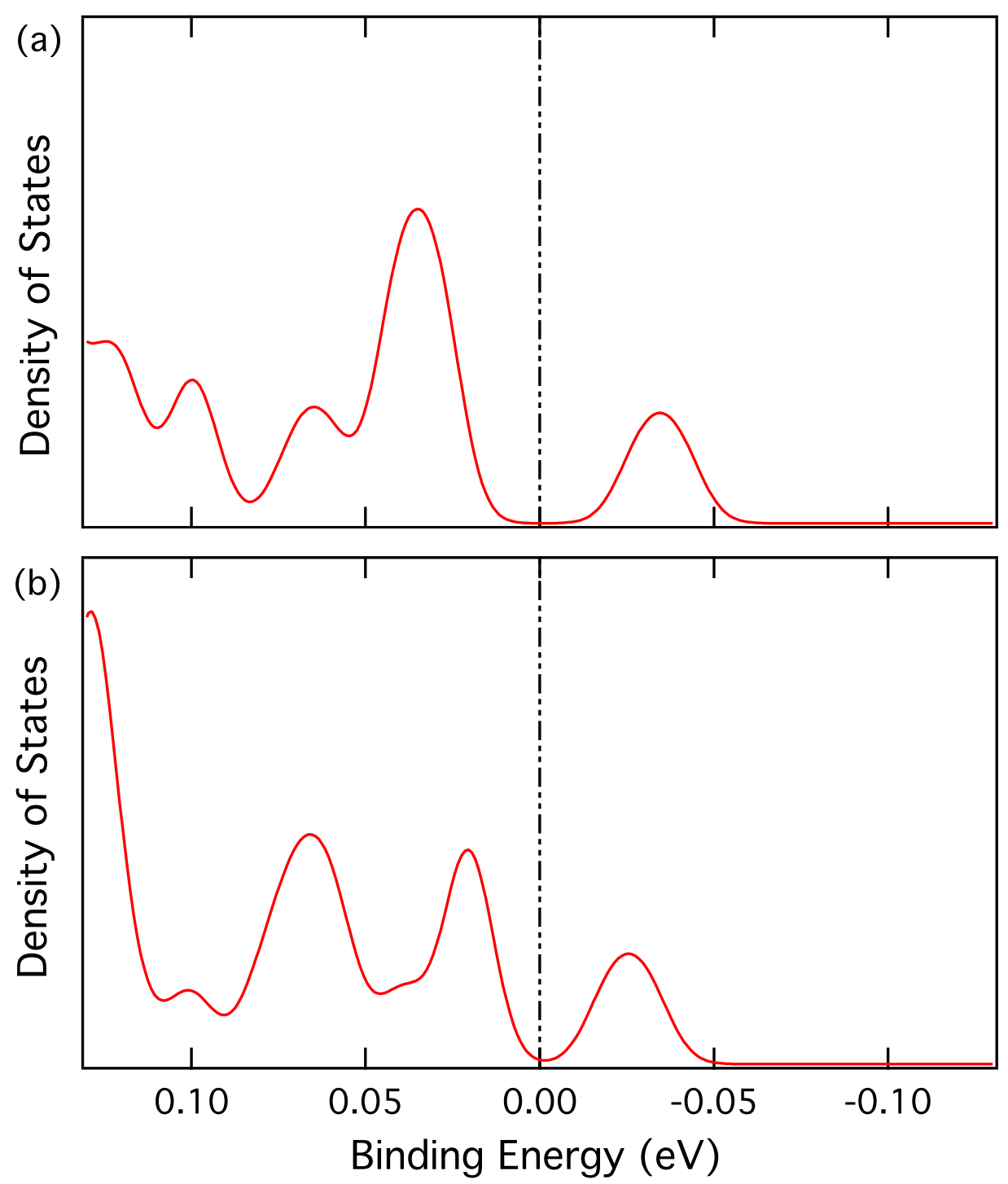

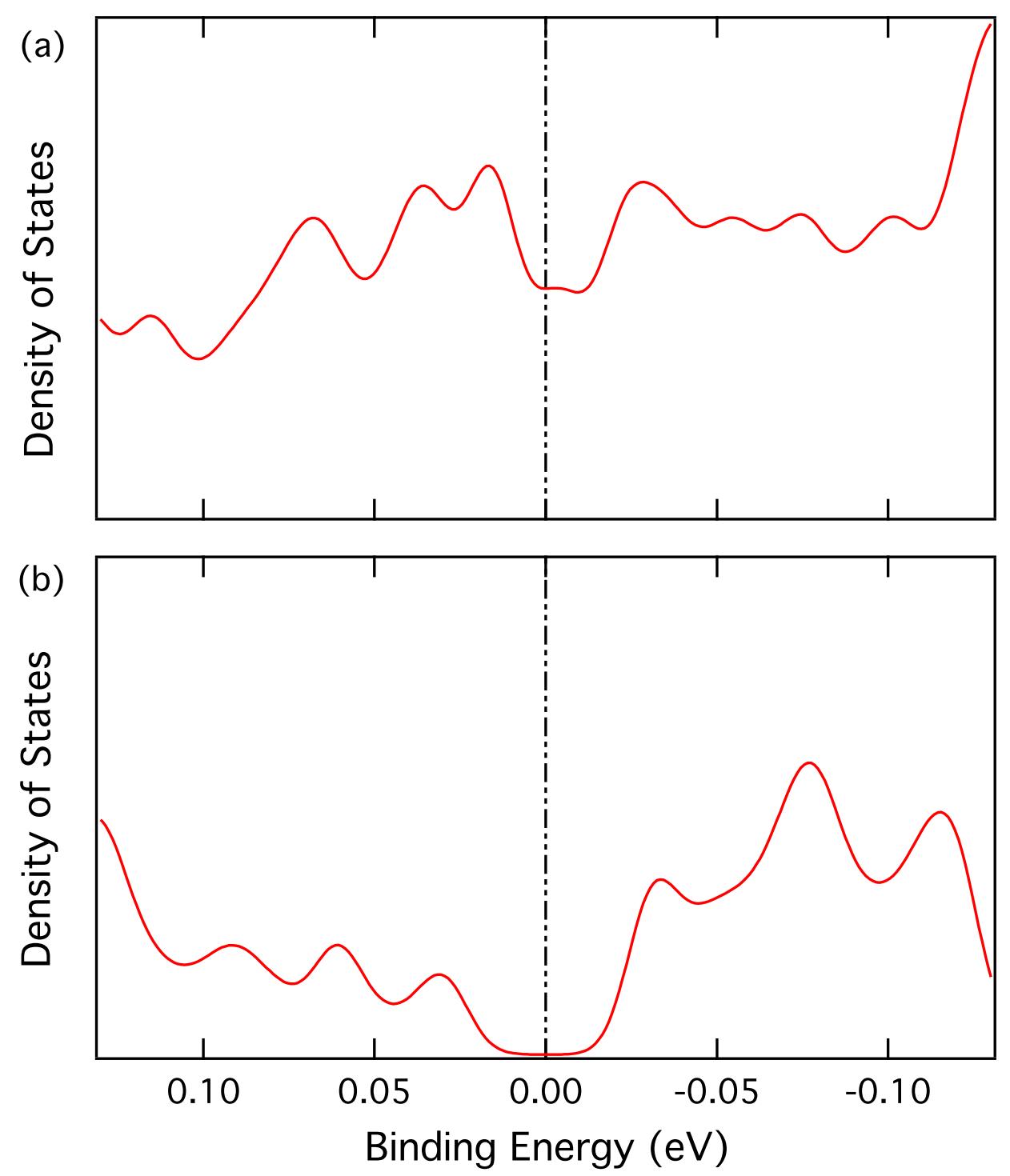